\title{
Outcome Indicator Matrix (OIM): a Tool to Assess National Action Plans
}

\section{Manuel Zometa}

Universidad de El Salvador, manuel.zometa2@ues.edu.sv

\begin{abstract}
The Outcome Indicator Matrix (OIM) (MIR in Spanish) is a tool that has been adapted by the Universidad de El Salvador (UES) to monitor commitments in national action plans (NAPs). It has been used to assess all commitments presented to the Open Government Partnership (OGP) by the Government of El Salvador that are in its NAP. The OIM-MIR is a result of integrating the logical framework method, used by many governments and public institutions and the focus of the Regulatory Impact Analysis (RIA) (OECD, 2018), which international organizations such as the OECD use to organize actions and procedures to produce a specific outcome.
\end{abstract}

Keywords: Open Government, National Action Plan, Outcome Indicators, Plan Assessment

\section{Introduction}

El Salvador has been a member of the Open Government Partnership (OGP, 2019) since 2011 and the University of El Salvador has been part of OGP El Salvador since 2013. As the only public university participating in OGP, UES plays a double role: first, to collaborate, participate, and cocreate El Salvador's NAPs as a member organization; and second, to comply with open government principles as a public institution. Likewise, the university sees its participation as an opportunity to test new tools and methods to improve NAPs' accomplishments and also to develop its own paradigm as an "open university."

The UES contributes to nongovernmental organizations (NGOs) and civil society in various ways, from technical support in research and data collection to report-building processes. In fact, the university drafts a report on every NAP that is presented to the Independent Reporting Mechanism of El Salvador (IRM), the government, and civil society. This report is created following OGP principles but using UES methods and tools such as the one explained in this text.

Currently, the Outcome Indicator Matrix (OIM-MIR) has been used by the UES to assess El Salvador's national action plans and commitments since 2014. It is a matrix of four rows and four columns in which the following data is presented: the objectives of the commitment and its indicators, the instruments that are created from the execution of the commitment or that are delivered as a result of it, the description of the indicators that measure the commitments' progress through the 
actions carried out for its achievement, the means of verification of each of the actions carried out and the information used to create the indicators, and finally, the elements that are beyond the reach of the institutions responsible for fulfilling the commitment.

\section{OIM-MIR Structure}

Identifying commitments' assessment variables through this matrix is done through a deductive logical scheme that allows government institutions and other committed organizations to identify each of the stages or actions taken while executing each commitment; this is generally done by describing such actions and its results. Once all data is gathered, an analysis can be performed by following a two-way causal relationship of the commitment's assessment and objectives.

The horizontal axis presents the commitment's monitoring and assessment, which make it possible to determine if it meets its objective. The columns address: a) narrative summary, which is a brief description of the action on every level; $b$ ) indicators, which describe evidence of the action taken; c) means of verification, to describe the instruments and documents used for each action or that show its result; and d) observations, which are the main adaptation made by the UES, and describe for further analysis the elements that are beyond the reach of institutions responsible for fulfilling the commitment. This last column indicates information sources for the indicators and goals, which allow observing and predicting the feasibility of the actions taken and the potential impact of the commitment; it also shows if all steps comply with the SMART principles proposed by OGP.

Table 1: Vertical Logic Analisys

\begin{tabular}{|l|l|l|l|l|}
\hline & Narrative Summary & Indicators & Means of verification & Observations \\
\hline & & & & \\
\hline & & & & $\longrightarrow$ \\
\hline & & & & $\longrightarrow$ \\
\hline & & & & $\longrightarrow$ \\
\hline
\end{tabular}

The vertical axis addresses the causal relations within the objective level of the commitment and provides evidence of the amount of actions to demonstrate whether there were too few or too many. The rows include: a) activities, where a brief description of initial actions aligns with the narrative summary column; b) components, as a result of the actions described on the line above, including documents or instruments created to achieve the commitment's goal; c) purpose, a brief description of how these documents, instruments, or tools will support the commitment's fulfillment, and d) goal, where the achieved or expected goal is described, logically aligned in a causal way. 
Table 2: Vertical Logic Analisys

\begin{tabular}{|c|c|c|c|c|}
\hline & Narrative Summary & Indicators & Means of verification & Observations \\
\hline Goal & 4 & 4 & 4 & $\uparrow$ \\
\hline Purpose & & & & \\
\hline Components & & & & \\
\hline Activities & & & & \\
\hline
\end{tabular}

\section{OIM-MIR Methodology}

The methodology used for observing and assessing outcome indicators from El Salvador's 2016-2018 action plan on the OIM-MIR clarifies the expected results of public programs, avoids all actions, tasks, instruments, or means of verification that do not contribute to the objective, and helps identify the necessary indicators to monitor the development and assessment of the whole plan.

Based on the Regulatory Impact Analysis parameters, the OIM-MIR analysis develops a reading scheme that builds a logical framework based on real, proven evidence of every action taken to achieve the commitment's goal. This analysis improves the design, organization, and monitoring of all the tasks and actions of commitments in action plans.

Within the matrix, it is necessary to identify the following data: a) the objectives of the commitment itself and their indicators; b) the changes, services, or policies created as a result of achieving the commitment's goal; c) the indicators that measure the commitment's impact through its actions; d) the means of verifying the actions and the information used to create these indicators; and e) the elements that are outside the scope of the institutions responsible for fulfilling the commitment.

Once the data is gathered, it must be analyzed following the causal logical relationship previously described, (horizontal and vertical) (CONEVAL, 2019) starting from the lower-left side of the table. The horizontal analysis should provide evidence of the elements of the commitment, as important parts of this assessment. Every column must contain an element that is described in the "Narrative Summary" and ends at the "Observations" column if anything prevented an element from being added. Analysis must be done, from left to right and it should end identifying the objective of the commitment, the purpose of the commitment's actions, and those elements that provide evidence of the elements necessary to carry out the objective.

Vertical analysis corresponds to the logical and sequential causality of each consecutive action, step, or task of a commitment. Reading should start from bottom of the table, moving upward to create a sequence of activities that leads to the final goal. Each of step of a commitment's achievement, from the first activity to the objective, is briefly described. From the beginning, the matrix provides a general overview of the commitment, then the indicators of the optimum amount of actions to solve the problem addressed by the commitment, and the risks it faces to fulfill its objective. 
Thus, the management of the activities established in the matrix, together with the formation of the assumptions, will allow the components to be carried out. Likewise, the delivery of public policies and services, coupled with the occurrence of the assumptions at the component level, will help to achieve the purpose, and so on.

It is important to understand that horizontal analysis works along the columns and the vertical analysis goes along the rows; this might be a bit confusing due to the tendency of reading columns from top to bottom. Although, conclusions should not vary it is possible to have slight variations in reading the matrix.

\section{Result Analysis and Feedback}

Analysis of the data in the OIM-MIR derives from the activities duly evidenced and whose contributions denote an unequivocal orientation toward achieving the indicator. The OIM-MIR specifically evaluates the logical execution of each commitment and the achievement of each indicator.

Results of this analysis, termed post-analysis data, are then presented in the form of statements, which objectively express what has been observed and assessed up to the analysis date. Interpretation of each statement is left up to the reader, thereby avoiding subjectivity. For this same reason, the quantitative evaluation is removed since the indicators and the method are eminently qualitative.

\section{References}

Consejo Nacional de Evaluación de la Politica de Desarrollo Social (CONEVAL). (2019). Homepage.

Retrieved September 18, 2019, from CONEVAL. (2019, septiembre 18).

https://www.coneval.org.mx/Paginas/principal.aspx. Retrieved from

https://www.coneval.org.mx/Paginas/principal.aspx

OECD. (2018, Septiembre 8). https://data.oecd.org/. Retrieved from https://data.oecd.org/

OGP. (2019, Septiembre). https://www.opengovpartnership.org/members/el-salvador/. Retrieved from https://www.opengovpartnership.org/members/el-salvador/

\section{About the Author}

Manuel Zometa

Professor of international law and public and government ethics at the Universidad de El Salvador, chief officer of the access to public information office (western campus), researcher of the scientific research secretariat of the same university on open government, technology, and law and civic participation. 